AperTO - Archivio Istituzionale Open Access dell'Università di Torino

\title{
Heuristic optimization of the scanning path of particle therapy beams
}

\section{This is the author's manuscript}

Original Citation:

\section{Availability:}

This version is available http://hdl.handle.net/2318/65337

since

Published version:

DOI:10.1118/1.3121506

Terms of use:

Open Access

Anyone can freely access the full text of works made available as "Open Access". Works made available under a Creative Commons license can be used according to the terms and conditions of said license. Use of all other works requires consent of the right holder (author or publisher) if not exempted from copyright protection by the applicable law. 


\section{(3) \\ UNIVERSITÀ DEGLI STUDI DI TORINO}

This is an author version of the contribution published on:

Medical Physics 36 (6) , June 2009, 2043

DOI:10.1118/1.3121506

The definitive version is available at:

http://scitation.aip.org/content/aapm/journal/medphys/ 


\title{
Heuristic optimization of the scanning path of particle therapy beams.
}

\author{
J Pardo,,${ }^{1, *}$ M Donetti, ${ }^{1,2}$ F Bourhaleb, ${ }^{3}$ A Ansarinejad, ${ }^{1,3}$ A Attili, ${ }^{1}$ R \\ Cirio, ${ }^{1,3}$ M A Garella, ${ }^{1}$ S Giordanengo, ${ }^{1}$ N Givehchi,,${ }^{1,3}$ A La Rosa, ${ }^{1,3, \dagger} \mathrm{F}$ \\ Marchetto, ${ }^{1}$ V Monaco, ${ }^{1,3}$ A Pecka, ${ }^{1,3}$ C Peroni,,${ }^{1,3}$ G Russo, ${ }^{1,3}$ and R Sacchi ${ }^{1,3}$ \\ ${ }^{1}$ Istituto Nazionale di Fisica Nucleare, Sezione di Torino, \\ Via P. Giuria 1, I-10125 Torino, Italy \\ ${ }^{2}$ Fondazione CNAO, Via Caminadella 16, I-20123, Milano, Italy \\ ${ }^{3}$ Dipartimento di Fisica Sperimentale, Università di Torino, \\ Via P. Giuria 1, I-10125 Torino, Italy
}

(Dated: March 24, 2009) 


\begin{abstract}
Purpose: Quasi-discrete scannig is a delivery strategy for proton and ion beam therapy in which the beam is turned off when a slice is finished and a new energy must be set, but not during the scanning between consecutive spots. Different scanning paths lead to different dose distributions due to the contribution of the unintended transit dose between spots. In this work an algorithm to optimize the scanning path for quasi-discrete scanned beams is presented.

Methods: The classical simulated annealing algorithm is used. It is a heuristic algorithm frequently used in combinatorial optimization problems, which allows to obtain nearly optimal solutions in acceptable running times.

Results: A study focused on the best choice of operational parameters on which the algorithm performance depends is presented. The convergence properties of the algorithm have been further improved by using the next-neighbour algorithm to generate the starting paths.

Scanning paths for two clinical treatments have been optimized. The optimized paths are found to be shorter than the back-and-forth, top-to-bottom (zigzag) paths generally provided by the treatment planning systems. The gamma method has been applied to quantify the improvement achieved on the dose distribution. Results show a reduction of the transit dose when the optimized paths are used. The benefit is clear especially when the fluence per spot is low, as in the case of repainting. The minimization of the transit dose can potentially allow the use of higher beam intensities, thus decreasing the treatment time.

Conclusions: The algorithm implemented for this work can optimize efficiently the scanning path of quasi-discrete scanned particle beams. Optimized scanning paths decrease the transit dose and lead to better dose distributions.
\end{abstract}

Keywords: Hadrontherapy, dose delivery, scanning beam, optimization

\footnotetext{
*Now at School of Cancer Studies, University of Liverpool, Liverpool, UK and Department of Physics, Clatterbridge Centre for Oncology, Wirral CH63 4JY, UK; Electronic address: juanpm@liv.ac.uk ${ }^{\dagger}$ Now at CERN, CH-1211, Switzerland
} 


\section{INTRODUCTION}

Proton and ion beams are particularly useful for radiotherapy applications due to their high dose localization (due to the Bragg peak) which improves the dose conformation to the tumour sparing the surrounding healthy tissues. In particle therapy with an active delivery system the target volume is divided into iso-energy slices, for every slice a set of positions (spots) is defined and each of these spots is irradiated by a pencil beam. The beam is steered between iso-energy spots using magnetic fields, while shifts in depth are performed by changing the beam energy. Two types of active scanning techniques are mainly used [1-3]: discrete or spot scan, where the beam is turned off during the scanning between consecutive spots, as at the Paul Scherrer Institute [4], and quasi-discrete scan, used at the Gesellschaft für Schwerionenforschung mbH [1], similar to the former case but where the beam is turned off only when a slice is finished and a new energy must be set.

Optimization of the scanning path for each slice is crucial for the quasi-discrete technique. In fact, the dose delivered during the transit between different spots (hereafter called transit dose), if not properly taken into account by the treatment planning system (TPS), affects the delivered dose distribution. This effect is particularly important when the transit time is comparable to the delivery time (irradiation time to deliver the planned number of particles

to a spot) [5]. The main goal of this work is to optimize the scanning path in order to reduce such effect. The optimization is less important when the spot scan technique is used because the beam is turned off after the irradiation of each spot. However, even in this case an optimized scanning path could help to reduce the treatment time and the energy consumption by the scanning magnets.

In this work the optimization of scanning paths like those present in spot scan or quasidiscrete delivery techniques is adressed. In a recent publication [6] Kang et al. have shown the utility of a heuristic algorithm, the fast simulated annealing, to optimize the scanning path, improve the dose conformation and reduce the treatment time (for a particular delivery strategy). In the present work a similar study based on the classical simulated annealing method [7] is presented. It is showed that the performance of the algorithm can be greatly improved when using an adequate set of operational parameters. The performance of the method is quantified by comparing the results with a simple scan where the spots in each slice are irradiated line-by-line in consecutive rows (zigzag path). Finally, the effect of an 
optimum scanning path on the delivered dose distribution and on the treatment delivery time is discussed.

The beam parameters and the beam delivery system considered in this work are those of the Centro Nazionale di Adroterapia Oncologica (CNAO) [8], currently under construction in Pavia, Italy. This center is a synchrotron-based facility which will treat patients with protons and carbon ions. A set of five fast-readout ionization chambers with different segmentation are used to monitor the beam position, shape and fluence, and provide fast feedback through a direct connection between the chamber readout and the magnet power supplies. When the measured fluence matches the prescribed one, the beam is shifted to the next spot without stopping the irradiation. Each spill covers a single slice and the beam intensity is not modulated during the spill.

\section{METHODS}

\section{A. The scanning path optimization}

The 3D scan path optimization can be naturally subdivided in a sequence of independent optimizations in each $2 \mathrm{D}$ slice $[6]$.

Let's consider a slice consisting of $N$ spots. Each spot position is defined by the transverse coordinates of its center $\left(x_{i}, y_{i}\right), i=1, \cdots, N$. The problem is to find the sequence in which the spots must be irradiated to minimize a given objective function. A scanning path, $P$, is a sequence of spots $P=\{\pi(1), \cdots, \pi(N)\}$, where $\pi(i)$ is the index identifying the spot that is irradiated in the $i$-th position of the sequence.

The objective function to minimize is the total transit dose delivered during the transition between spots. Considering a constant intensity beam, this is equivalent to minimize the transit time between spots. In the approximation of a constant scanning speed equal for the $\mathrm{X}$ and $\mathrm{Y}$ magnets (at CNAO the design relative difference between horizontal and vertical scanning speeds is of the order of 10\%), the transition time between two spots $i$ and $j$ is

proportional to $\max \left(\left|x_{i}-x_{j}\right|,\left|y_{i}-y_{j}\right|\right)$, leading to the following objective function of the scanning path:

$$
g(P)=\sum_{i=1}^{N-1} \max \left(\left|x_{\pi(i)}-x_{\pi(i+1)}\right|,\left|y_{\pi(i)}-y_{\pi(i+1)}\right|\right)
$$


However, in this function diagonal displacements are equivalent to horizontal or vertical displacements, whichever is larger. This might lead to several crossings in the path, as shown in figure 1(A), which should be avoided because of the potential creation of hot spots. The use of the following objective function based on the Euclidean distance

$$
f(P)=\sum_{i=1}^{N-1}\left[\left(x_{\pi(i)}-x_{\pi(i+1)}\right)^{2}+\left(y_{\pi(i)}-y_{\pi(i+1)}\right)^{2}\right]^{1 / 2}
$$

penalizes diagonal movements, potentially avoiding crossings in the scanning path as shown in figure 1(B). As both functions lead to similar results but the Euclidian one avoids crossings, only function (2) is used in the following.

\section{B. The simulated annealing algorithm}

The minimization of equation (2) is a combinatorial problem which needs a large computation time to obtain the exact solution. The simulated annealing (SA) method [7] is one of the most useful heuristic algorithms for this kind of optimization problems which guarantees the convergence to a near-optimal solution in a reasonable time. It has been used in several fields where combinatorial optimization problems are present such as the travelling salesman problem [10]. It has been frequently used in the field of radiotherapy, for example to optimize treatment dose distributions [11].

The SA algorithm is based on an analogy with the way liquids freeze and crystallize or metals cool and anneal. In the SA algorithm the objective function $f$ is the equivalent of the energy and a control parameter $T$ plays the role of the temperature. At fixed temperature, the algorithm generates random perturbations of the system leading to new configurations (equilibrium loop). The system is set in the new configuration if this corresponds to a lower value of the objective function (downhill movement), while if it results in a higher value, the new configuration is accepted with a probability depending on the temperature (uphill movement). Uphill movements are introduced to allow escaping from local minima. When the equilibrium is approached, the temperature is decreased and the equilibrium loop is repeated for a new value of the temperature (cooling loop). 


\section{Implementation of the algorithm}

The SA algorithm for the minimization of the objective function (2) has been implemented in $\mathrm{C}++$. The schematic operation of the algorithm is as follows:

1. Generate (or read) a starting path $P_{0}$ and evaluate the objective function, $f\left(P_{0}\right)$. Set a starting temperature $T_{0}$ (expressed in units of length).

2. Start Cooling loop.

3. Start Equilibrium loop.

(3.1) Obtain a new path $P$ by performing a random rearrangement of $P_{0}$

(3.2) Compute $\Delta=f(P)-f\left(P_{0}\right)$

(3.3) If $\Delta \leq 0$ (downhill move) set $P_{0}=P$

(3.4) Else, choose a random number $r \in[0,1]$. If $r<e^{-\Delta / T}$ set $P_{0}=P$ (uphill move)

4. End Equilibrium loop: go to 3 if Equilibrium loop is not finished.

5. Decrease the temperature.

6. End Cooling loop: go to 2 if Cooling loop is not finished.

7. Run finished: return $P_{0}$.

Within this work the length of the equilibrium loop is set to $100 \times N$, which has been checked to be long enough to guarantee a reasonable approach to the equilibrium. The rearrangement of the path can be performed in several ways. In this work we have implemented two operations (following [9]): (a) a path segment of size $n$ (i.e. connecting $n$ spots) is removed from the path and replaced with the same segment in reverse order; (b) a path segment of size $n$ is removed and inserted in another region. A random number is used to select with equal probability the operation that will be performed for each iteration of the equilibrium loop. 
The temperature parameter determines the survival probability of an uphill movement. At each step $i$ of the cooling loop the temperature parameter is defined as

$$
T_{i}=a^{i} T_{0}
$$

where $a$, in the range $(0,1)$, is the cooling parameter which controls the rate of the temperature decrease.

\section{RESULTS AND DICUSSION}

\section{A. Optimization of the algorithm parameters}

The performance of this algorithm strongly depends on the operational parameters, especially on the size $n$ of the rearrangement segment and on the cooling parameter $a$. Implementation of the algorithm without selecting a good set of operational parameters can lead to a slow convergence and poor results. A study of its performance for several parameter values has been performed in order to find adequate operational conditions. Several slices have been used for this study in order to guarantee that the selection of a good set of operational parameters does not strongly depend on the considered set of spots. For the sake of simplicity results are shown just for a slice if not stated otherwise: a head-and-neck treatment that contains 129 spots distributed in a $66 \mathrm{~mm} \times 72 \mathrm{~mm}$ area (shown in figure $6(\mathrm{E})-(\mathrm{F}))$. For these studies the initial value of the temperature parameter has been set to $T_{0}=0.5 \mathrm{~mm}$.

\section{SA algorithm performance: size of the rearrangement segment}

Two strategies regarding the size $n$ of the path segment rearranged at each iteration have been studied: constant or variable size. Figure $2(\mathrm{~A})$ shows the results obtained when a constant size is used $(n=3,20$ or 50$)$. The evolution of the total length of the scanning path is shown as a function of the number of equilibrium loop iterations. A random starting path has been considered and the equilibrium loop has been repeated 20 times in order to determine the average path length shown in the figure (standard deviations are not shown in the figure for the sake of simplicity). A faster convergence is obtained when the size of 
the rearrangement segment is a random integer number. Figure 2(B) shows the results for a size choosen randomly in the ranges $n \in[1,0.5 N], n \in[1,0.75 N]$ and $n \in[1, N]$.

Figure 3 shows the path length as a function of the number of cooling loop iterations for the different choices of segment size defined above. The cooling parameter has been set to $a=0.9$, the cooling loop has been repeated 20 times and the average value and standard deviation of the path length are shown. The choice of a fixed size for the path segment used for the rearrangement is clearly disfavoured, as can be seen comparing figure 3(A) and (B). Moreover it is observed in figure 3(B) that a faster convergence is obtained when the segment size $n$ is sampled in a wider interval.

These features can be explained observing that a variable segment size allows for both small and large modifications of the path length; small modifications are effective in the proximity of the minimum while large changes are effective to accelerate the convergence in the initial steps and to escape from local minima. From now on, only a variable size of the rearrangment segment in the interval $[1, N]$ will be considered.

\section{SA algorithm performance: initial temperature and cooling parameter}

Figure 4 shows the length of the scanning path as a function of the number of cooling loop iterations for several values of the cooling parameter $(a=0.5, a=0.7$ and $a=0.9$ ). As above, the cooling loop has been repeated 20 times in order to determine the average path length and the standard deviation for each iteration. In this example there are three main local minima, with $775.45 \mathrm{~mm}, 778.94 \mathrm{~mm}$ and $781.85 \mathrm{~mm}$ total length. If a too fast cooling is used (see figure $4(\mathrm{~A})$ ) the convergence to a local minimum is difficult to avoid. On the other hand, it has been observed that a too slow cooling $(a>0.9)$ results in a too slow convergence because the survival probability for uphill movements is large even after a large number of iterations. The optimal compromise is found to be $a=0.9$.

\section{SA algorithm performance: combination with the nearest neighbour method}

An effective way to improve the efficiency of the method is to accelerate its convergence through the use of a path close to the optimum solution as the starting path. Two options have been tried: the use of the simple zigzag path and the use of a path computed with 
the nearest neighbour (NN) algorithm. The NN algorithm consists in building a path by selecting an arbitrary starting spot and by recursively connecting spots to the path, the next being the nearest unordered spot to the last spot added. This process is repeated by changing the starting spot and the path with the minimum length among all the possibilities is chosen.

Figure 5 shows the convergence of the SA algorithm when using the zigzag and the solution of the NN algorithm as the initial paths. Results are shown in figure $5(\mathrm{~A})$ for the slice containing 129 spots of figure 6(E), and in figure 5(B) for a slice consisting of 100 spots (the slice is shown in figure $7(\mathrm{E})$ ). The convergence is faster when the NN algorithm is used to create the initial path, especially in the latter case where the difference in length between the path returned by the NN algorithm and the zigzag path is larger.

\section{B. Optimization of the scanning path for clinical treatments}

The SA method has been applied to optimize the scanning path for several clinical cases. Taking into account the results of the previous section, the cooling parameter $a$ is set to 0.9 and the size $n$ of the rearranged segment is chosen randomly in the range $[1, N]$. The initial path is computed with the NN algorithm and the value of the initial temperature parameter $T_{0}$ is set to allow a significant survival probability for the first iterations. The cooling loop is stopped if 5 consecutive iterations do not improve the scanning path or after 50 iterations.

Two clinical treatments obtained with the Syngo PT planning system have been used: a head-and-neck treatment consisting of 877 spots distributed in 11 slices with a grid spacing of $6 \mathrm{~mm}$ and a chondrosarcoma treatment consisting of 6892 spots distributed in 38 slices with a grid spacing of $3 \mathrm{~mm}$. The scanning paths obtained with our optimization algorithm have been compared with those provided by the TPS (a zigzag path).

The optimization of the head-and-neck treatment results in path lengths between $2 \%$ and $18 \%$ shorter than the zigzag paths in each slice, with a reduction of $8 \%$ for the whole treatment. Respectively, for the chondrosarcoma treatment the reduction is between $1 \%$ and $10 \%$ for each slice, with a global reduction of $5 \%$. Figure 6 compares the optimized and zigzag scanning paths for three slices of the head-and-neck treatment. It can be observed that the optimized paths avoid the irradiation of holes or regions surrounding the treatment area. 
The slices of these treatments are quite compact, meaning that they consist of a single cluster of spots. As already shown in [6], the length reduction and the conformation is more significant when the optimization is performed for slices with several separated clusters of spots. The same slices used in [6] have been optimized with our algorithm. Figure 7 shows the scanning paths obtained. The length reduction, compared to the zigzag path, is $31.6 \%, 23.4 \%, 35.3 \%, 33.0 \%, 56.2 \%$ and $65.4 \%$ for the slices (A) to (F) shown in the figure. Compared with the results reported in [6], the path lengths are $9.4 \%, 10.0 \%, 14.4 \%, 15.4 \%$, $4.3 \%$ and $9.1 \%$ shorter. As both algorithms are similar, the better results are attributed to the careful selection of the algorithm operational parameters (see section III A). In addition, scanning paths obtained with the algorithm presented in this work do not contain intersections which could in principle lead to dose overshooting as discussed in section II A.

\section{Dosimetric implications}

In order to study the effect of the scanning path on the dose distribution of specific treatments, several parameters such as the beam intensity and the scanning speed must be defined. In the following the specifications of the CNAO active beam delivery system are considered. At CNAO the beam is dynamically steered along the scanning path without intensity modulation. When the planned number of particles has been delivered to a spot, the beam is shifted to the next spot without stopping the irradiation. The particles delivered during the shift are assigned by the beam delivery system to the destination spot. This method ensures that the total number of particles delivered to each slice matches the planned one. However, the transit dose may affect the dose distribution. This effect will be studied assuming a constant scanning speed at the CNAO design value of $20 \mathrm{~m} \mathrm{~s}^{-1}$.

The quantitative effect of the scanning path on the dose distribution must be studied in three dimensions (3D), because the beam associated to a given spot does not only contribute to the dose of that slice, but also delivers dose to the more proximal slices. However, a twodimensional (2D) sketch, considering just a single slice, can be useful to understand the effect of a specific scanning path on the particle distribution in the slice. 


\section{A two-dimensional example}

For the 2D analysis the optimized and zigzag scanning paths shown in figure 6 (C) and (D) for a slice of the head-and-neck treatment are used. A beam with a constant intensity of $4 \times 10^{8}$ particles $\mathrm{s}^{-1}$, corresponding to the CNAO maximum intensity for a carbon ion beam, delivers $10^{6}$ particles to each spot of the slice. The beam full width at half maximum (FWHM) is 3 times larger than the distance between neighbour spots to ensure a homegeneous distribution.

Figure 8 shows the comparison between the reference distribution of particles, evaluated assuming a null transit time between spots, and those obtained with the zigzag path and the optimized path. The distributions are evaluated on a grid with $2 \mathrm{~mm} \times 2 \mathrm{~mm}$ pixel size. The relative differences with respect to the reference distribution normalized to the plateau region are also evaluated in each pixel of the grid and shown in the figure. The 2D particle distribution obtained with the zigzag path differs up to $4 \%$ from the reference one. The difference is especially important in the top part of the slice, where the zigzag path crosses a region not belonging to the slice. The optimized path avoids this region and allows to deliver a distribution very similar to the reference one, with local relative differences smaller than $0.5 \%$.

\section{Three-dimensional dose distribution}

In order to evaluate the $3 \mathrm{D}$ dose distributions the position of the spots, the number of particles delivered to each spot, the beam energy and its FWHM have been taken from the TPS output for both the head-and-neck and the chondrosarcoma treatments. The dose deposition in depth, including the effect of the beam broadening, has been taken from lookup tables obtained from Monte Carlo simulations performed with GEANT3 [12]. The dose distribution has been obtained by steering the beam along the scanning path, both for the zigzag and the optimized one, and has been compared with the reference distribution evaluated assuming a null transit time between spots. Beam intensities of $10^{10}, 5 \times 10^{9}$ and $10^{9}$ protons $\mathrm{s}^{-1}$, typical values for the CNAO proton beam, have been used. The dose distributions have been computed in a water-equivalent medium using a voxel size of $2 \mathrm{~mm}$ $\times 2 \mathrm{~mm} \times 2 \mathrm{~mm}$. 
The gamma method [13] has been used to compare the reference dose distributions with those obtained with the optimized and zigzag scanning paths. For each voxel $i$ of the non-reference dose distribution (that computed using the zigzag or optimized paths), $\gamma_{i}$ is computed as

$$
\gamma_{i}=\min _{j}\left(\sqrt{\frac{d_{i j}^{2}}{d_{0}^{2}}+\frac{\Delta D_{i j}^{2}}{\Delta D_{0}^{2}}}\right)
$$

where $d_{i j}$ is the euclidean distance between voxel $i$ of the non-reference distribution and voxel $j$ of the reference distribution, $\Delta D_{i j}$ is the dose difference between the same voxels relative to the reference dose in the spread-out Bragg peak region. $d_{0}$ and $\Delta D_{0}$ are the tolerance values: distance-to-agreement and dose difference (we use $3 \mathrm{~mm}$ and $3 \%$ respectively).

The distribution of $\gamma$ values for the chondrosarcoma treatment is shown in figure 9 for the highest beam intensity. Table I shows a summary of the results for different beam intensities and for the two treatments. The discrepances with respect to the reference distributions are evaluated in terms of the average value of $\gamma$ and percentage of voxels with $\gamma<1$. In addition, the maximum local difference (relative difference normalized to the dose in the spread out bragg peak region), $\Delta D^{\max }$, calculated averaging the dose distributions on voxels of size 1 $\mathrm{cm} \times 1 \mathrm{~cm} \times 1 \mathrm{~cm}$, is reported in the same table. The latter quantity is more sensitive to local disomogenities than the percentage of voxels with $\gamma<1$.

When the beam intensity is low all the voxels have $\gamma<1$ for both the optimized and zigzag paths, nonetheless the average value of $\gamma$ is lower for the optimized path. For larger beam intensities the percentage of points with $\gamma<1$ decreases for the zigzag distribution and is always very close to $100 \%$ for the optimized path. The maximum local differences $\Delta D^{\max }$ are up to $5 \%$ for the zigzag distributions, and a factor 2-4 lower when using the optimized paths. These results point out the superiority of optimized scanning paths over conventional zigzag paths.

\section{The effect of repainting}

In clinical practice the interplay between scanning and target movement can considerably degrade the delivered dose distributions. Repainting is one of the techniques used to mitigate the effect of intrafractional motions [14]. In the simplest scenario it consists on delivering 
the prescribed number of particles per slice by repeating the scanning path $m$ times, where $m$ is the repainting factor, thus reducing in each painting the number of particles delivered at each spot by the same factor.

The reduction of the number of particles per spot causes an increase of the transit time to delivery time ratio. Usually this ratio is small, e.g. $\sim 0.1$ for a transit time around $100 \mu \mathrm{s}$ and a delivery time around $1 \mathrm{~ms}$. With a large repainting factor the ratio increases, eventually approaching 1 in some steps, a limit value for which the whole number of particles would be delivered during the shift between spots. The benefit of an optimized scanning path is therefore crucial for large repainting factors, for which the effect of the transit dose on the dose distribution can become sizeable.

The 3D dose distribution of the chondrosarcoma treatment has been recalculated for both the optimized and zigzag scanning paths, as described in the previous section, considering repainting factors of 5 and 10. The $\gamma$-test was performed and the average values of $\gamma$, the percentages of voxels with $\gamma<1$ and the maximum local differences $\Delta D^{\max }$ are shown in table II. The differences between the delivered and reference distributions are larger than those calculated with a single painting reported in table I, and increase with the beam

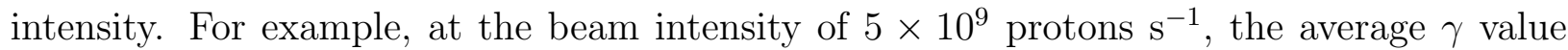
with the optimized path increases from 0.04 (no repainting) to 0.37 (repainting factor $m=5$ ) and $0.65(m=10)$. The benefit of using an optimized path is observed in the maximum local difference. For example, for $m=5, \Delta D^{\max }$ is $1.6 \%$ in contrast to a value of $24.7 \%$ obtained with the zigzag paths. From these results it can be concluded that, at the typical values of the CNAO beam intensities, the dose distribution obtained with the optimized scanning path, in contrast with the zigzag path, matches reasonably well the reference dose even for large repainting factors.

\section{Impact on the treatment time}

As shown in the previous sections, the effect of the transit dose strongly depends on the beam intensity. In order to reduce this effect the beam intensity should be reduced, at the cost of increasing the treatment time. Alternatively, a similar improvement could be achieved by optimizing the scanning path without decreasing the beam intensity.

For example, for the chondrosarcoma treatment the quality of the dose distribution ob- 
tained with no repainting is quite similar for an intensity of $I=10^{10}$ protons $\mathrm{s}^{-1}$ and an

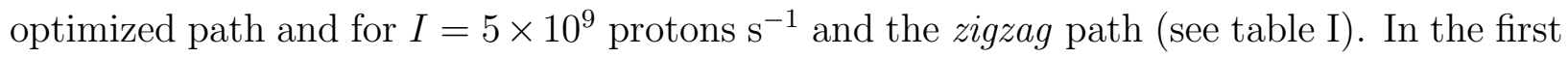
case the global treatment time is approximately $10 \%$ shorter (40 s instead of $44 \mathrm{~s}$ ). This reduction is not larger because most of the treatment time is spent in uploading the spills and switching the beam energy (around $1 \mathrm{~s}$ is needed to upload a new spill at CNAO).

In contrast to synchrotron-based facilities, the energy-switch time is shorter in cyclotrons. Thus, the reduction of the treatment time due to the implementation of optimized paths would be potentially larger for cyclotron facilities. Considering a value of $200 \mathrm{~ms}$ to switch the beam energy (typical value for a cyclotron), the treatment time reduction in the above example would be around $25 \%$.

\section{Running time}

An important issue to be considered in an optimization problem is its running time. For the SA algorithm presented, the running time strongly depends on the number of slices and spots of the treatment. In the present work the algorithm has been implemented in $\mathrm{C}++$, and run in a AMD Athlon X2 at $2.4 \mathrm{GHz}$. For the chondrosarcoma treatment, consisting of 38 slices and 6892 spots, the running time is around 6 minutes, an acceptable time for the clinical practice. The optimization of the scanning path for the head-and-neck treatment (877 spots, 11 slices) required only 0.3 minutes.

\section{CONCLUSIONS}

An algorithm based on the classical simulated annealing method has been developed to optimize the scanning path of actively delivered hadrontherapy beams. The performance of the algorithm has been studied in order to obtain a good set of operational parameters,

allowing to achieve the optimal solution with a high probability and after a relatively small number of iterations. Furthermore, the use of a starting path obtained with the nearestneighbour algorithm allows to reduce the number of iterations needed for the convergence. The running time of the implemented algorithm is found to be acceptable for a clinical application.

The SA algorithm provides scanning paths significantly shorter than simple zigzag paths, 
with a length reduction depending on the slice geometry. For compact slices the length reduction is around a few per cent, while for slices containing disperse clusters of spots the improvement can be more than $50 \%$. Scanning paths obtained with this algorithm have been compared with those obtained using a similar algorithm recently developed [6]. Slightly better results are obtained with our implementation, probably due to the careful selection of the algorithm operational parameters.

The effect of the scanning path on the dose distribution has been studied for two proton treatments using the specifications of the beam delivery system of CNAO. Dose distributions obtained with the optimized and zigzag scanning paths have been compared with the reference dose distribution using a 3D $\gamma$-test. The best agreement with the reference dose distribution is obtained using the optimized path, for which the contribution of the transit dose is reduced. The improvement relative to a zigzag scanning path is more evident when repainting is considered.

Even for a non optimized path, the effect of the transit dose can be reduced by decreasing the beam intensity, at the cost of increasing the treatment time. However, treatments provided with an optimized path can achieve the same agreement with the reference distribution without decreasing the beam intensity.

\section{Acknowledgments}

We would like to thank Dr. Fabrizio Banci Buonamici for providing us the clinical treatments used in this work. 
[1] T. Haberer, W. Becher, D. Schardt, and G. Kraft, Magnetic scanning system for heavy ion therapy, Nucl. Instrum. and Methods A 330, 296 (1993).

[2] T. Furukawa, T. Inaniwa, S. Sato, T. Tomitani, S. Minohara, K. Noda, and T. Kanai, Design study of a raster scanning system for moving target irradiation in heavy-ion radiotherapy, Med. Phys. 34, 1085 (2007).

[3] P. M. DeLuca, A. Wambersie, and G. Whitmore, Beam delivery and properties, Journal of the ICRU 7 7, 29 (2007).

[4] E. Pedroni, R. Bacher, H. Blattmann, T. Böhringer, A. Coray, A. Lomax, S. Lin, G. Munkel, S. Scheib, U. Schneider, and A. Tourovsky, The $200-\mathrm{MeV}$ proton therapy project at the Paul Scherrer Institute: Conceptual design and practical realization, Med. Phys. 22, 37 (1995).

[5] T. Inaniwa, T. Furukawa, T. Tomitani, S. Sato, K. Noda, and T. Kanai, Optimization for fast-scanning irradiation in particle therapy, Med. Phys. 34, 3302 (2007).

[6] J. H. Kang, J. J. Wilkens, and U. Oelfke, Demonstration of scan path optimization in proton therapy, Med. Phys. 34, 3457 (2007).

[7] S. Kirkpatrick, C. D. Gelatt, and M. P. Vecchi, Optimization by simulated annealing, Science 220, 670 (1983).

[8] S. Giordanengo et al, The CNAO system to monitor and control hadron beams for therapy, IEEE Dresden, Nuclear Science Symposium - Conference Record, 5609 (2008).

[9] V. T. Vetterling, W. H. Press, S. A. Teukolsky, and B. P. Flannery, Numerical Recipes Example Book $(C++)$ : The Art of Scientific Computing (Cambridge University Press, 2002).

[10] D. S. Johnson and L. A. McGeoch, The traveling salesman problem: a case study in local optimization, in Local Search in Combinatorial Optimization (John Willey and Sons, 1997).

[11] S. Webb, Optimisation of conformal radiotherapy dose distribution by simulated annealing, Phys. Med. Biol. 34, 1349 (1989).

[12] R. Brun, F. Bruyant, M. Maire, A. C. McPherson, and P. Zanarini, GEANT 3, Technical Report CERN-DD/EE/84-1, (1987).

[13] D. A. Low, W. B. Harms, S. Mutic, and J. A. Purdy, A technique for the quantitative evaluation of dose distributions, Med. Phys. 25, 656 (1998).

[14] M. H. Phillips, E. Pedroni, H. Blattmann, T. Behringer, A. Coray, and S. Scheib, Effects of 
respiratory motion on dose uniformity with a charged particle scanning method, Phys. Med. Biol. 37, 223 (1992). 

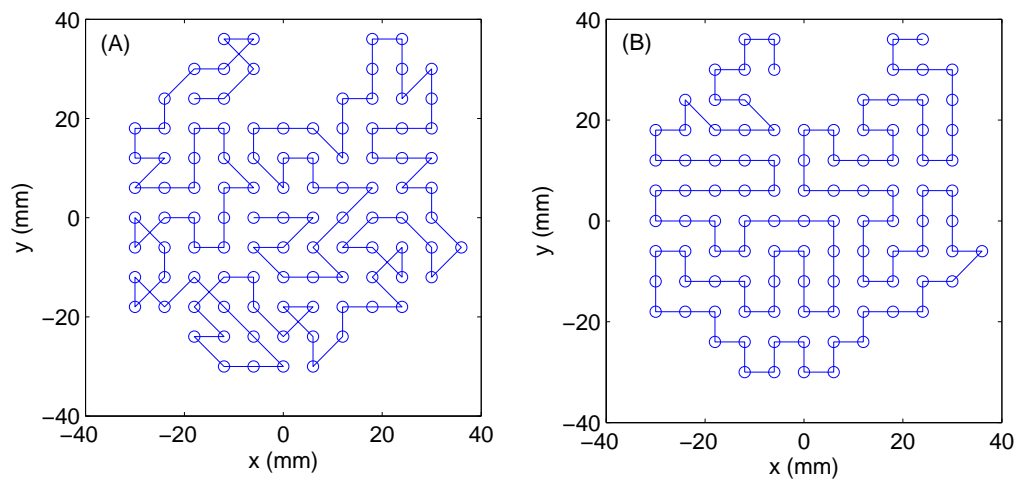

FIG. 1: Optimized scanning path for a typical slice obtained by minimizing (A) equation (1) and (B) equation (2). Crossings in the scanning path are clearly apreciated in the former case.
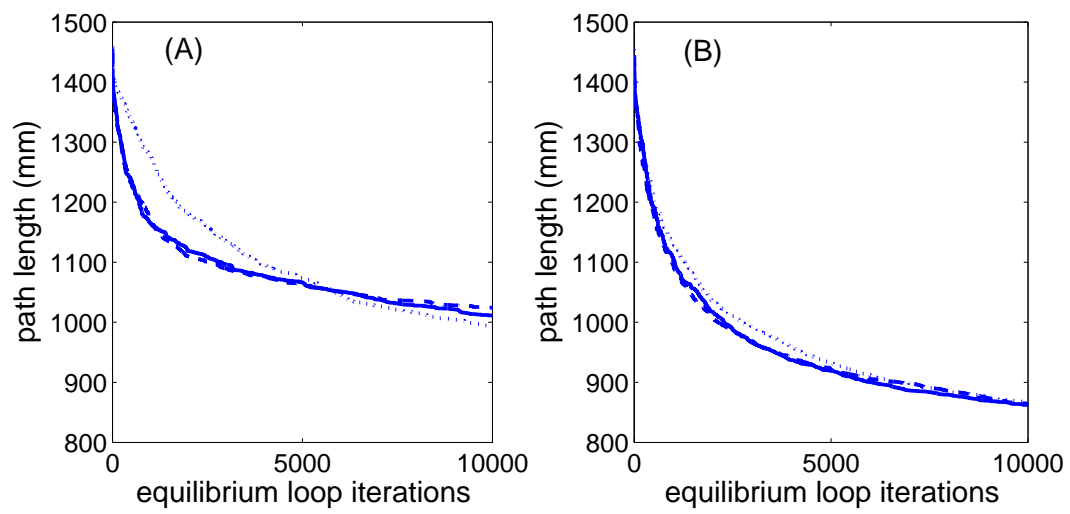

FIG. 2: Convergence of the SA method within the equilibrium loop for different values of the rearrangement segment size: (A) constant length, 3 (dotted line), 20 (dashed line) and 50 (solid line) spots; (B) variable length, $n \in[1,0.5 N]$ (dotted line), $n \in[1,0.75 N]$ (dashed line) and $n \in[1, N]$ (solid line). Data show the average value of 20 runs. 

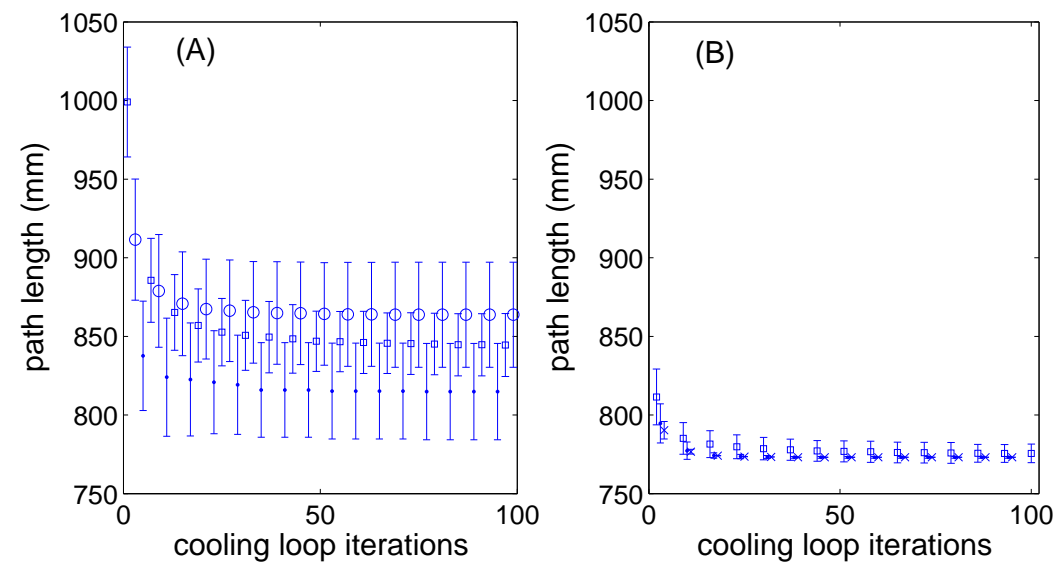

FIG. 3: Convergence of the SA method within the cooling loop for different values of the rearrangement segment size: (A) constant length, $3(\square), 20($ ( ) and $50(\cdot)$ spots; (B) variable length, $n \in[1,0.5 N](\square), n \in[1,0.75 N](\cdot)$ and $n \in[1, N](\times)$. Data show the average value and the standard deviation (error bar) of 20 runs.
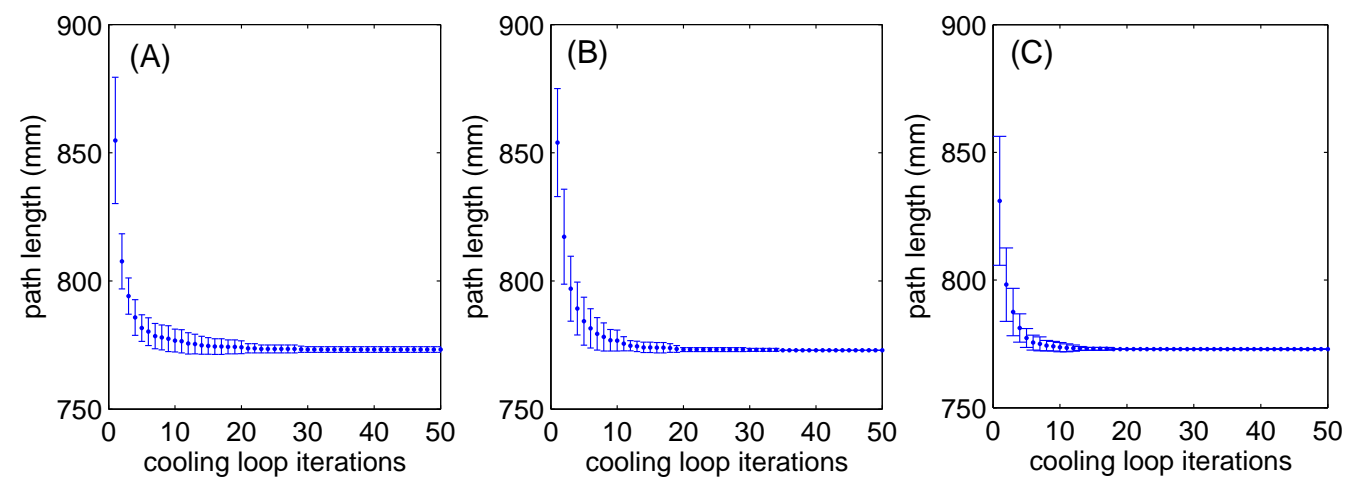

FIG. 4: Convergence of the SA method for different values of the cooling parameter: (A) a=0.5, (B) $\mathrm{a}=0.7,(\mathrm{C}) \mathrm{a}=0.9$. Data show the average value and the standard deviation (error bar) of 20 runs. 

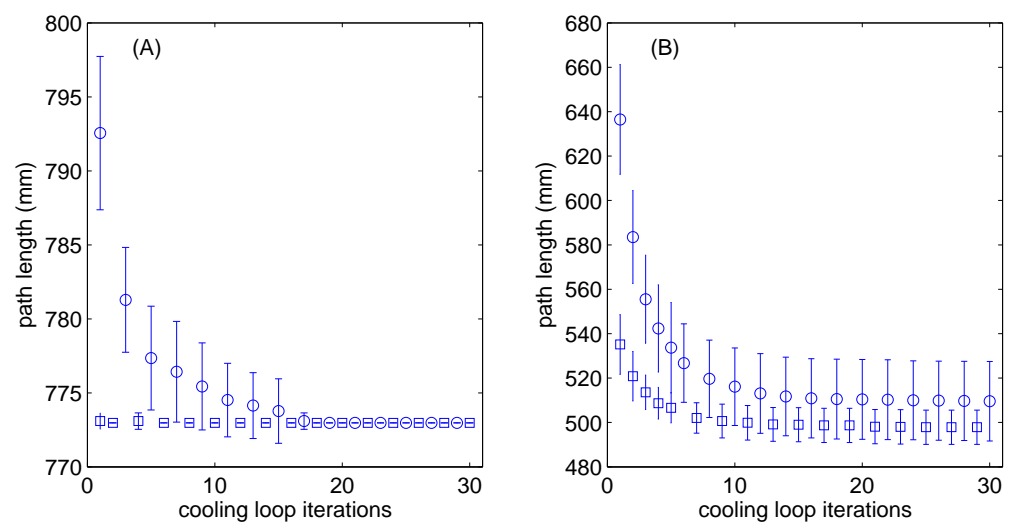

FIG. 5: Convergence of the SA method applied to two slices, one with 129 spots (A) and the other one with 100 (B), when using a starting path created with the NN algorithm ( $\square$ ) or the zigzag path (o). Data show the average value and the standard deviation (error bar) of 20 repetitions of the cooling loop.

TABLE I: Comparison (average $\gamma$, percentage of points with $\gamma<1$ and relative maximum local difference in a $1 \mathrm{~cm}^{3}$ volume) between the reference dose distribution and those obtained using optimized $\left(\bar{\gamma}_{\mathrm{opt}}, P_{\mathrm{opt}}, \Delta D_{\mathrm{opt}}^{\max }\right)$ and zigzag paths $\left(\bar{\gamma}_{\mathrm{zz}}, P_{\mathrm{zz}}, \Delta D_{\mathrm{zz}}^{\max }\right)$ for different beam intensity values.

\begin{tabular}{|c|c|c|c|c|c|c|}
\hline \hline \multicolumn{7}{|c|}{ Chondrosarcoma treatment } \\
\hline \hline intensity $\left(\mathrm{s}^{-1}\right)$ & $\bar{\gamma}_{\mathrm{opt}}$ & $\bar{\gamma}_{\mathrm{zz}}$ & $P_{\mathrm{opt}}(\%)$ & $P_{\mathrm{zz}}(\%)$ & $\Delta D_{\mathrm{opt}}^{\max }(\%)$ & $\Delta D_{\mathrm{zz}}^{\max }(\%)$ \\
\hline $10^{10}$ & 0.10 & 0.21 & 100 & 99.4 & 0.6 & 2.5 \\
$5 \times 10^{9}$ & 0.04 & 0.08 & 100 & 100 & 0.3 & 1.1 \\
$10^{9}$ & 0.008 & 0.02 & 100 & 100 & 0.05 & 0.4 \\
\hline \hline \multicolumn{7}{|c|}{ Head-and-neck treatment } \\
\hline \hline intensity $\left(\mathrm{s}^{-1}\right)$ & $\bar{\gamma}_{\mathrm{opt}}$ & $\bar{\gamma}_{\mathrm{zz}}$ & $P_{\mathrm{opt}}(\%)$ & $P_{\mathrm{zz}}(\%)$ & $\Delta D_{\mathrm{opt}}^{\max }(\%)$ & $\Delta D_{\mathrm{zz}}^{\max }(\%)$ \\
\hline $10^{10}$ & 0.14 & 0.26 & 99.9 & 97.8 & 2.3 & 4.9 \\
$5 \times 10^{9}$ & 0.07 & 0.14 & 100 & 99.9 & 1.0 & 2.6 \\
$10^{9}$ & 0.01 & 0.03 & 100 & 100 & 0.2 & 0.5 \\
\hline
\end{tabular}



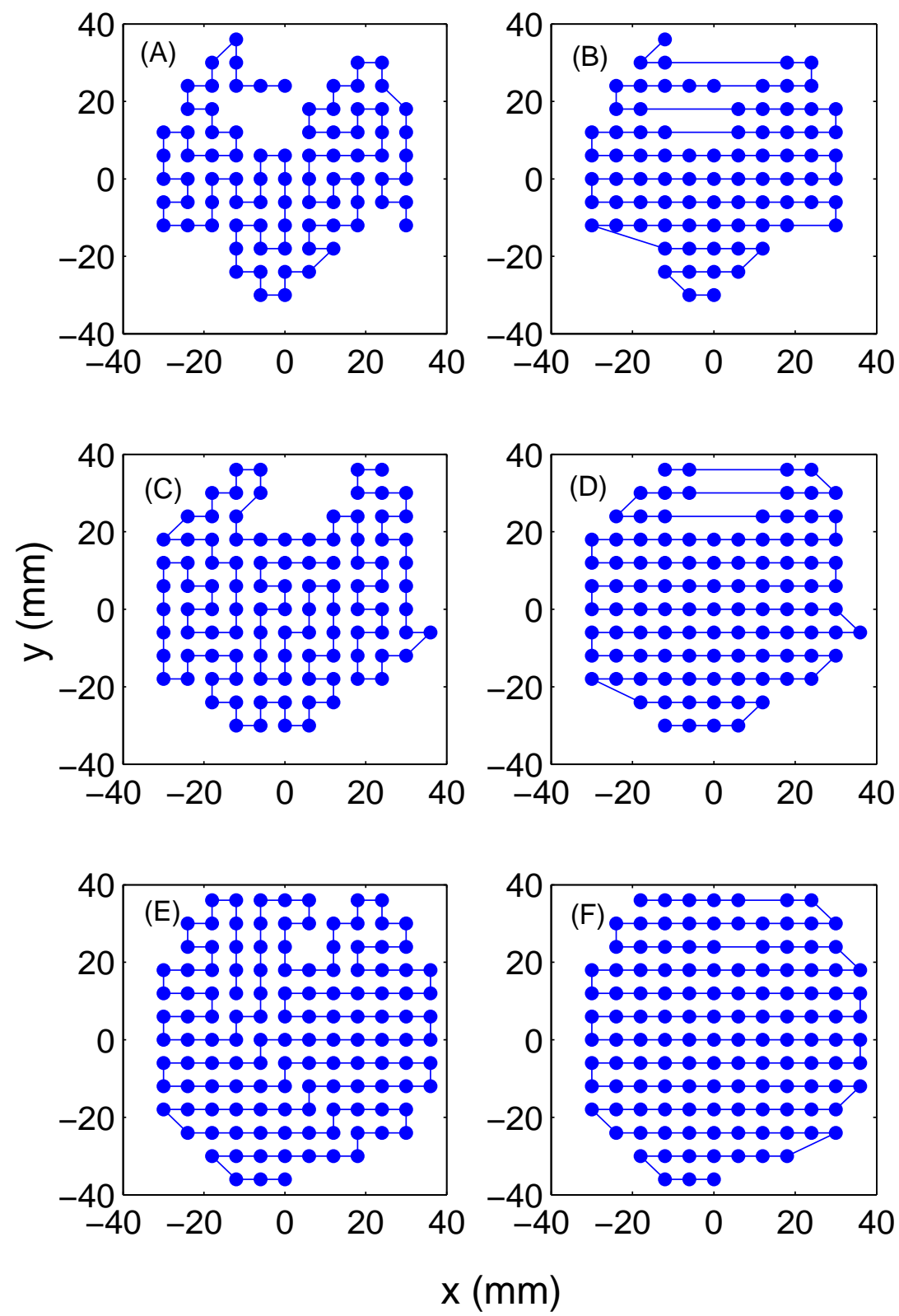

FIG. 6: Optimized (left panels) and zigzag (right panels) scanning paths for three slices of a head-and-neck treatment. 

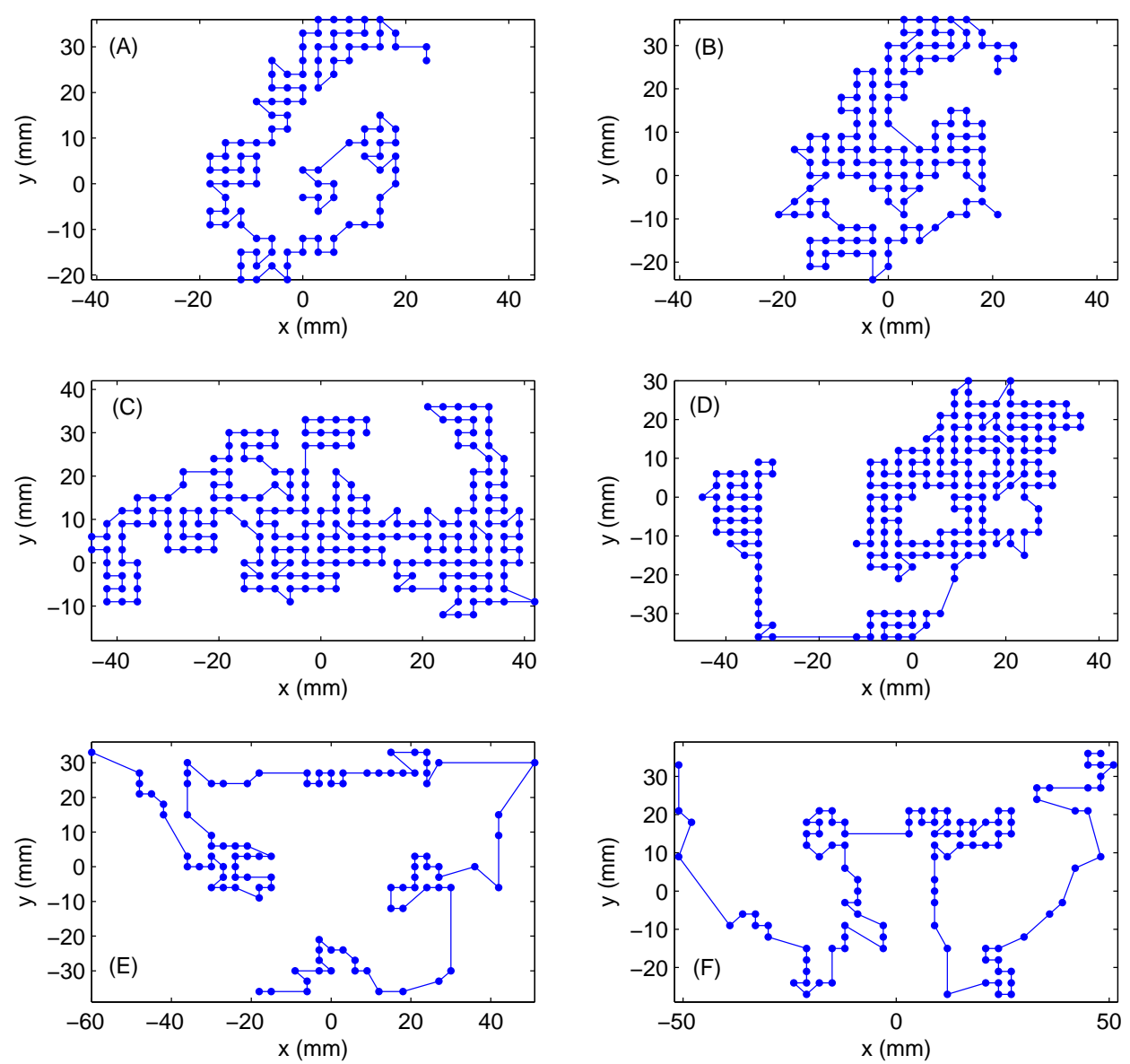

FIG. 7: Optimized scanning paths for the slices taken from [6]. 

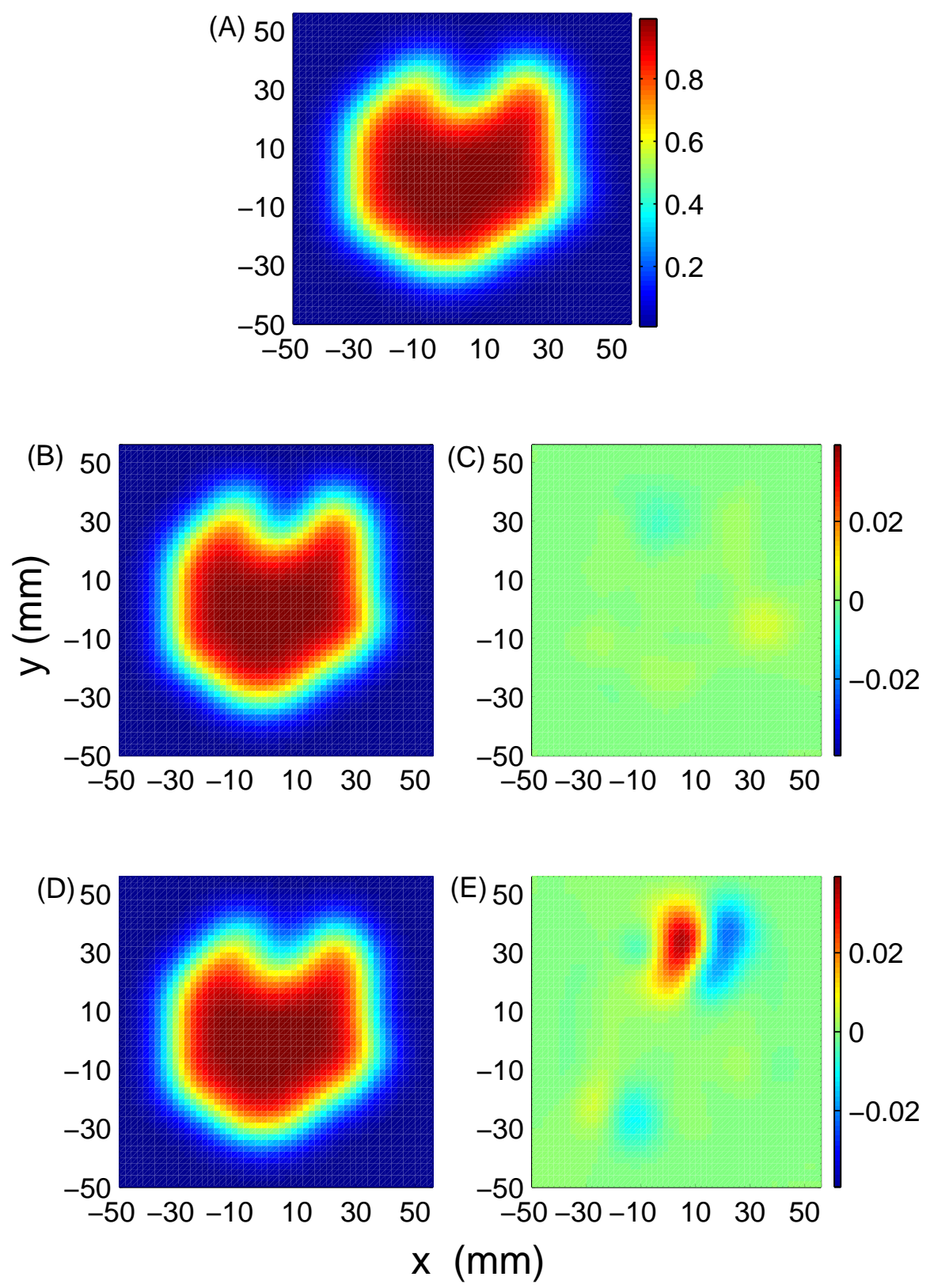

FIG. 8: Particle distribution for a slice: (A) reference distribution; (B) distribution obtained with the optimized scanning path showed in figure $6(\mathrm{C}) ;(\mathrm{C})$ relative difference normalized to the plateau region between the reference and delivered distributions using the optimized path; (D) distribution obtained with the zigzag path showed in figure 6(D); (E) relative difference normalized to the plateau region between the reference and delivered distributions using the zigzag path. 


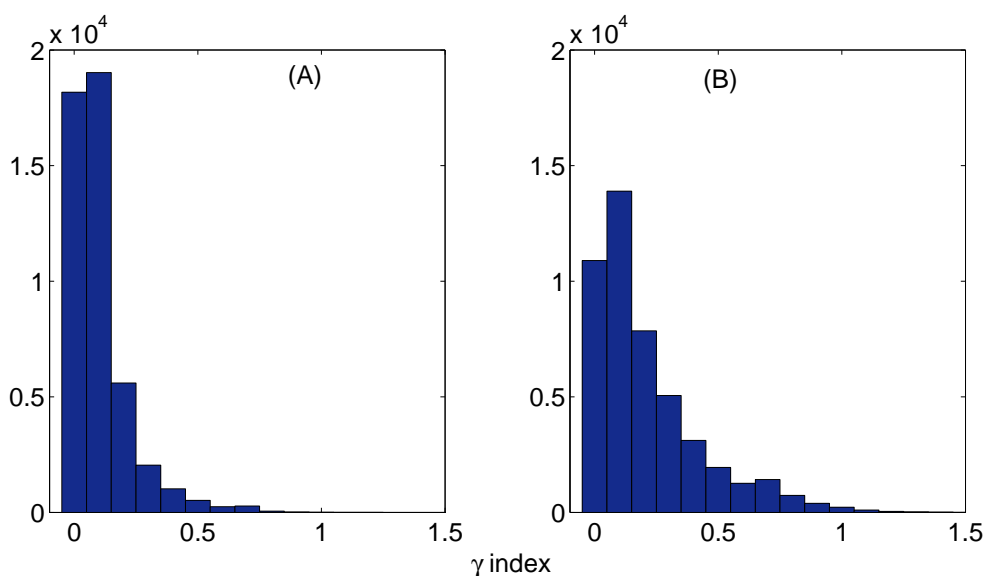

FIG. 9: Histogram of the $\gamma$-index for the chondrosarcoma treatment obtained comparing the reference dose distribution and those obtained using optimized (A) and zigzag (B) scanning paths. The beam intensity is $10^{10}$ protons $\mathrm{s}^{-1}$.

TABLE II: Comparison (average $\gamma$, percentage of points with $\gamma<1$ and relative maximum local difference in a $1 \mathrm{~cm}^{3}$ volume) between the reference dose distribution and those obtained using optimized $\left(\bar{\gamma}_{\mathrm{opt}}, P_{\mathrm{opt}}, \Delta D_{\mathrm{opt}}^{\max }\right)$ and zigzag paths $\left(\bar{\gamma}_{\mathrm{zz}}, P_{\mathrm{zz}}, \Delta D_{\mathrm{zz}}^{\max }\right)$ for different beam intensity values and repainting factor 5 and 10. Results are for the chondrosarcoma treatment.

\begin{tabular}{|c|c|c|c|c|c|c|}
\hline \hline \multicolumn{7}{|c|}{ Repainting factor $=10$} \\
\hline \hline intensity $\left(\mathrm{s}^{-1}\right)$ & $\bar{\gamma}_{\mathrm{opt}}$ & $\bar{\gamma}_{\mathrm{zz}}$ & $P_{\mathrm{opt}}(\%)$ & $P_{\mathrm{zz}}(\%)$ & $\Delta D_{\mathrm{opt}}^{\max }(\%)$ & $\Delta D_{\mathrm{zz}}^{\max }(\%)$ \\
\hline $5 \times 10^{9}$ & 0.65 & 1.28 & 83.8 & 44.8 & 7.9 & 56.4 \\
$10^{9}$ & 0.10 & 0.21 & 100 & 99.4 & 0.6 & 2.5 \\
\hline \hline \multicolumn{7}{|c|}{ Repainting factor $=5$} \\
\hline \hline intensity $\left(\mathrm{s}^{-1}\right)$ & $\bar{\gamma}_{\mathrm{opt}}$ & $\bar{\gamma}_{\mathrm{zz}}$ & $P_{\mathrm{opt}}(\%)$ & $P_{\mathrm{zz}}(\%)$ & $\Delta D_{\mathrm{opt}}^{\max }(\%)$ & $\Delta D_{\mathrm{zz}}^{\max }(\%)$ \\
\hline $5 \times 10^{9}$ & 0.37 & 0.88 & 96.6 & 65.0 & 1.6 & 24.7 \\
$10^{9}$ & 0.04 & 0.08 & 100 & 100 & 0.3 & 1.1 \\
\hline
\end{tabular}



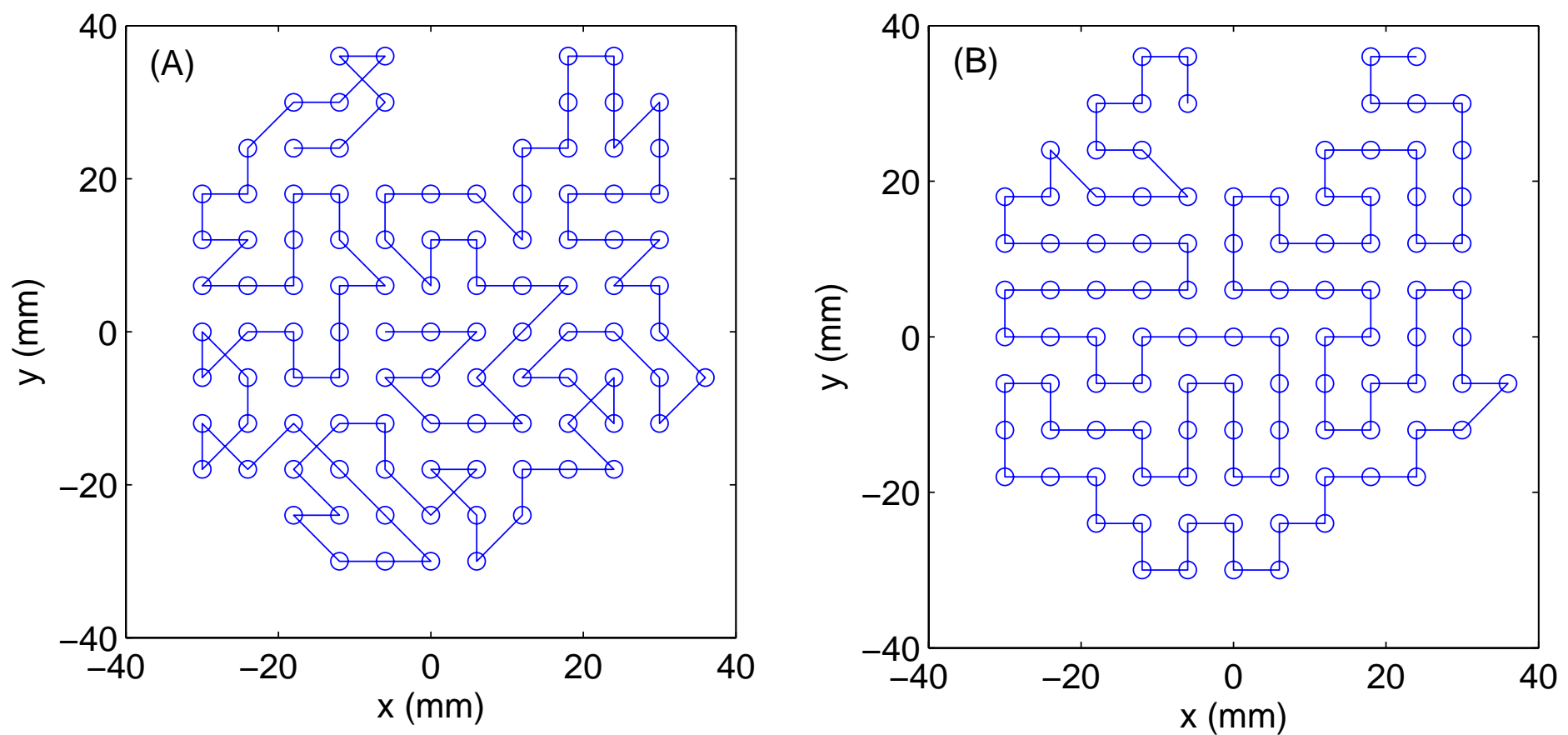

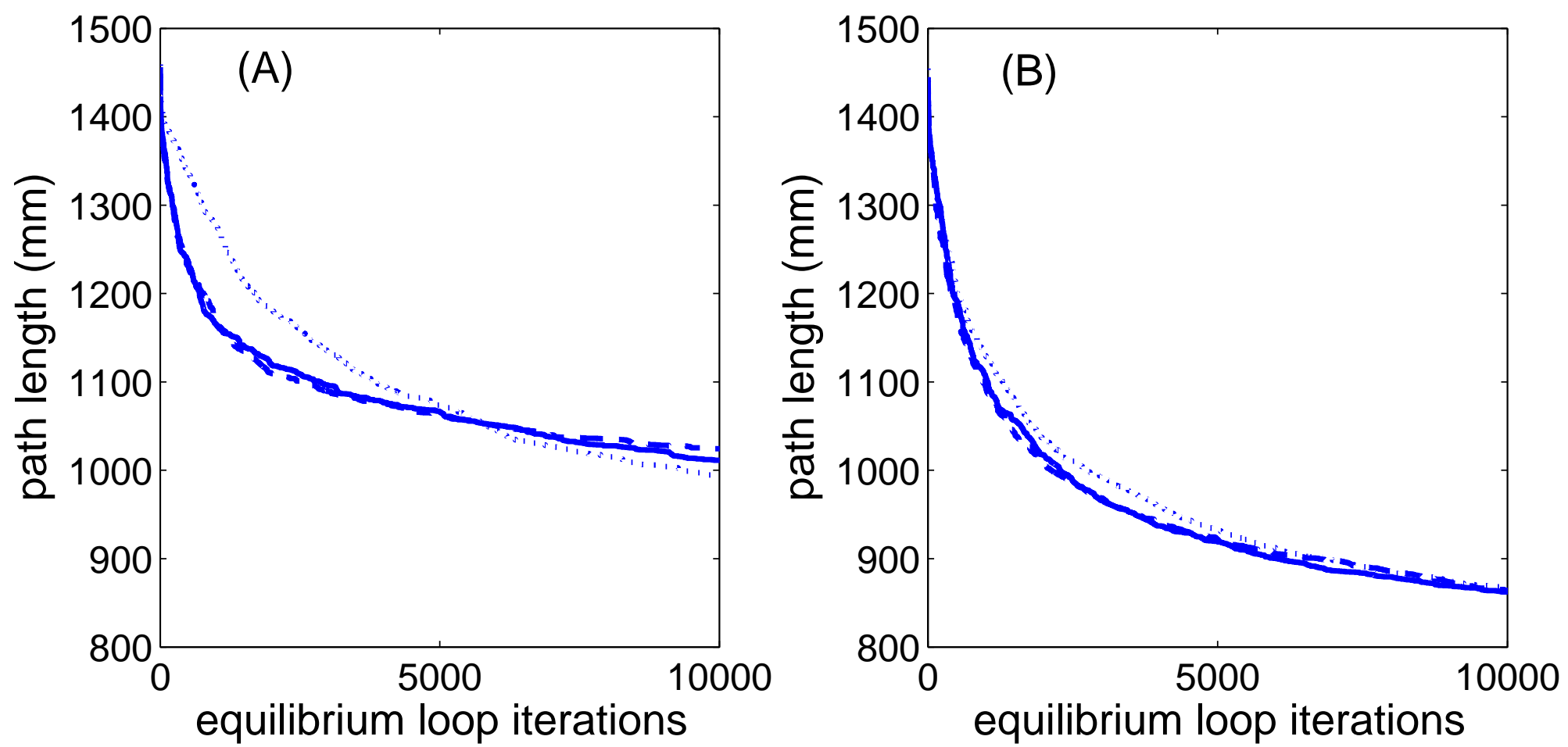

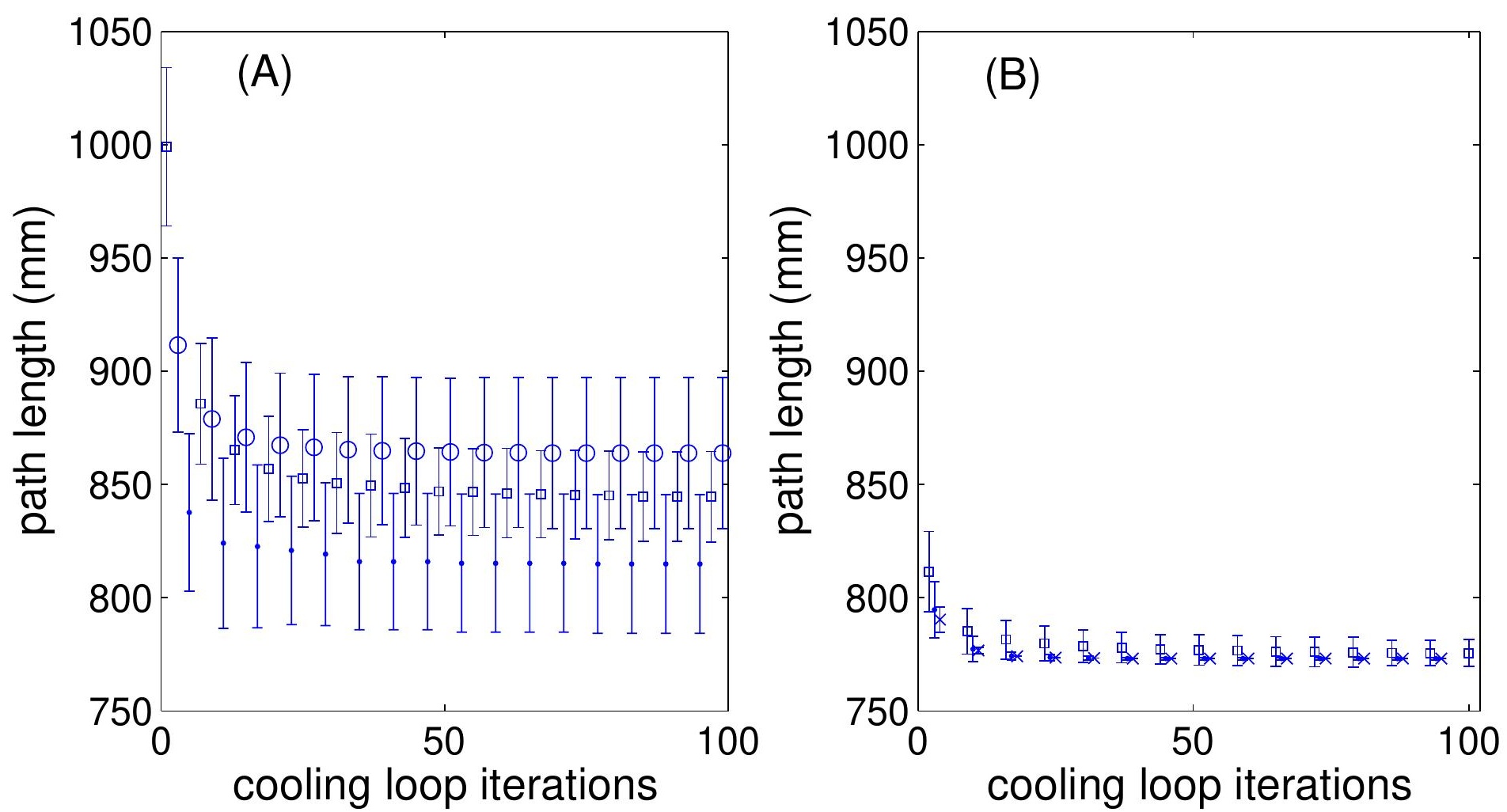

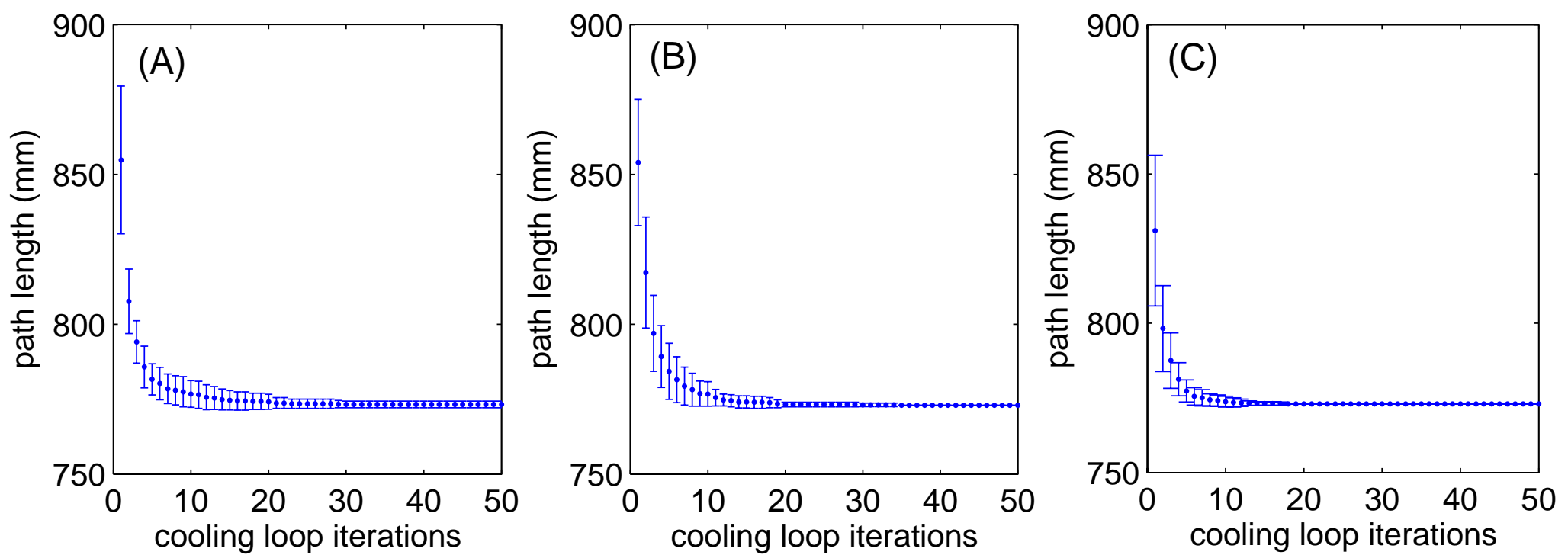

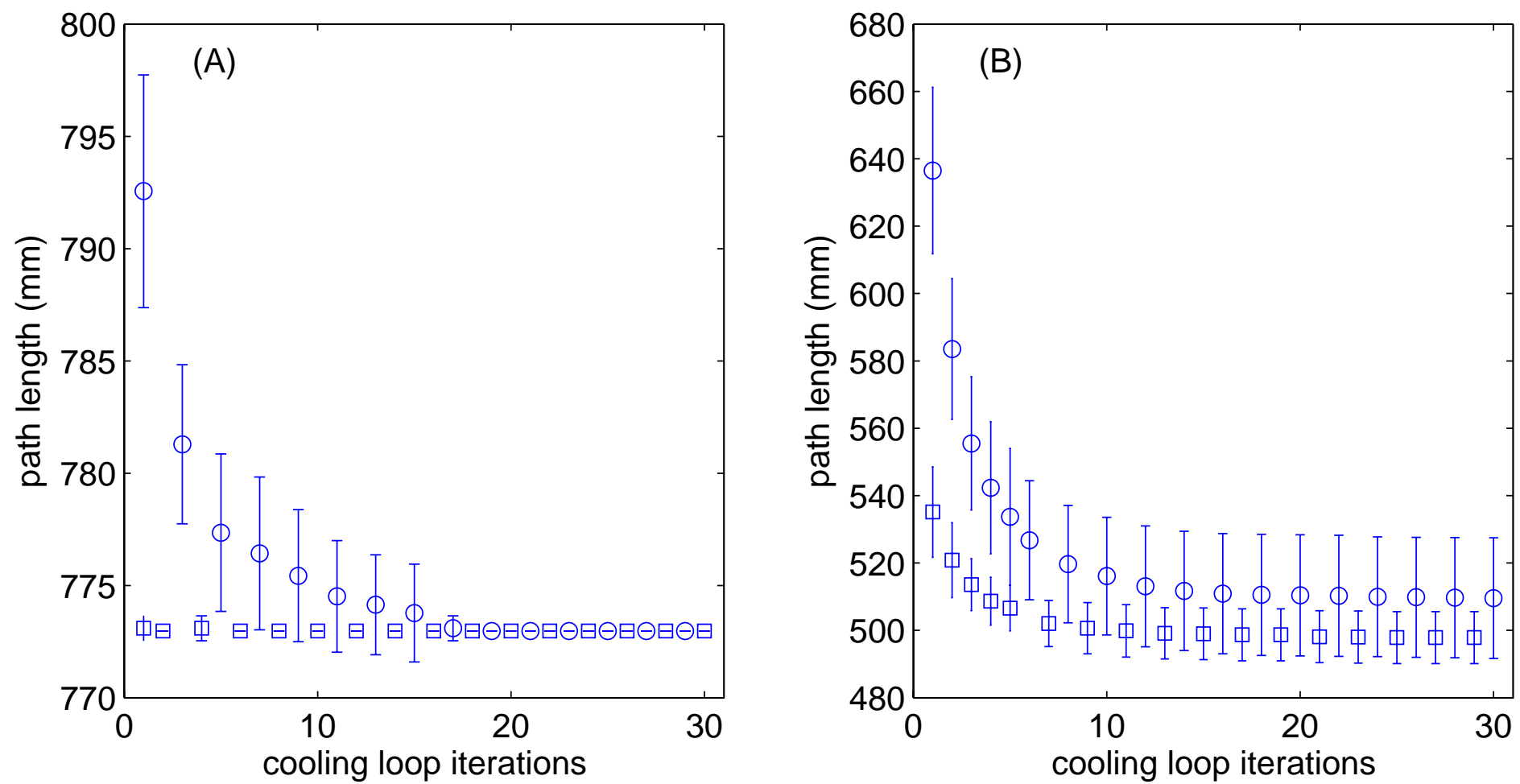

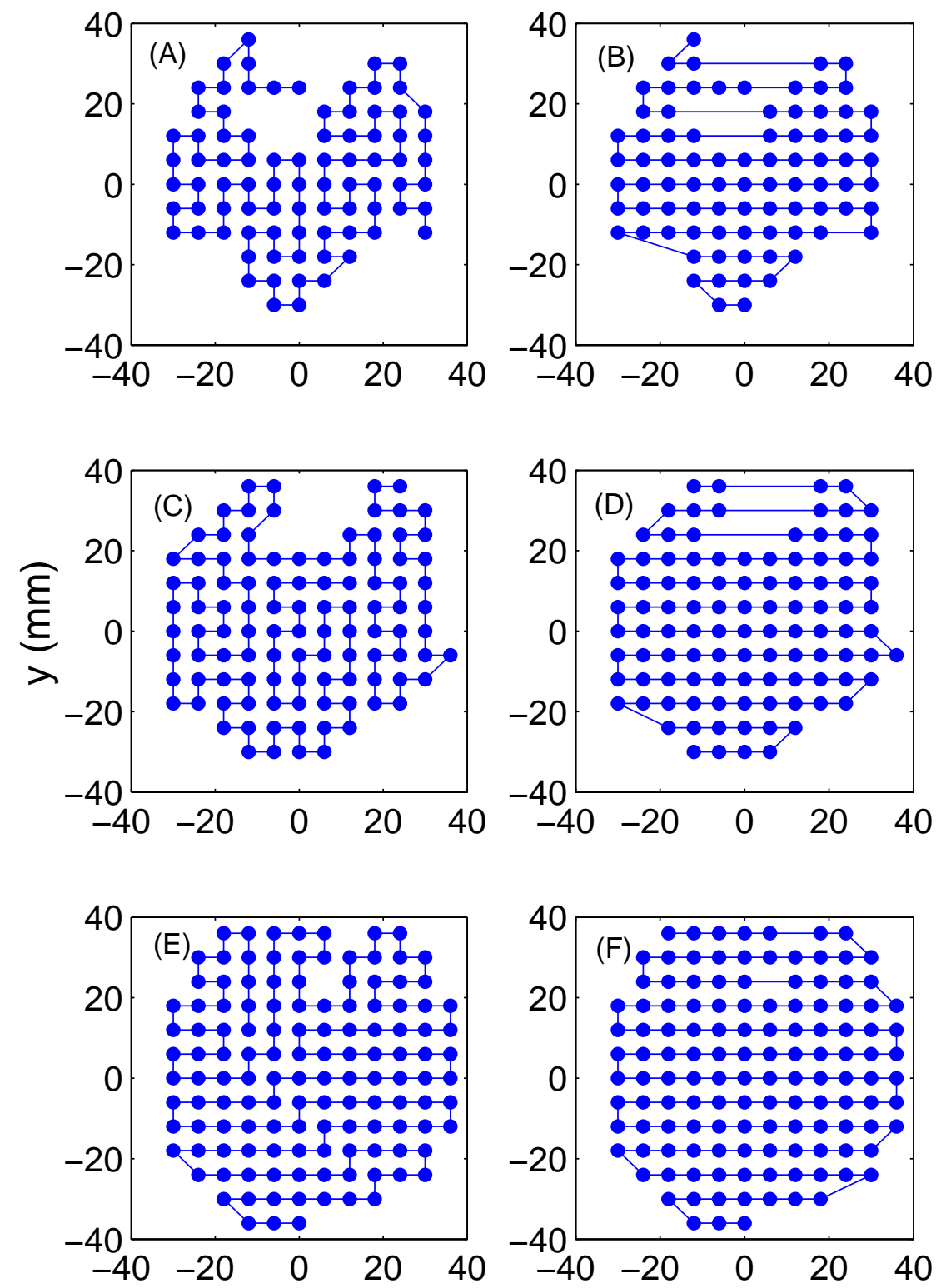

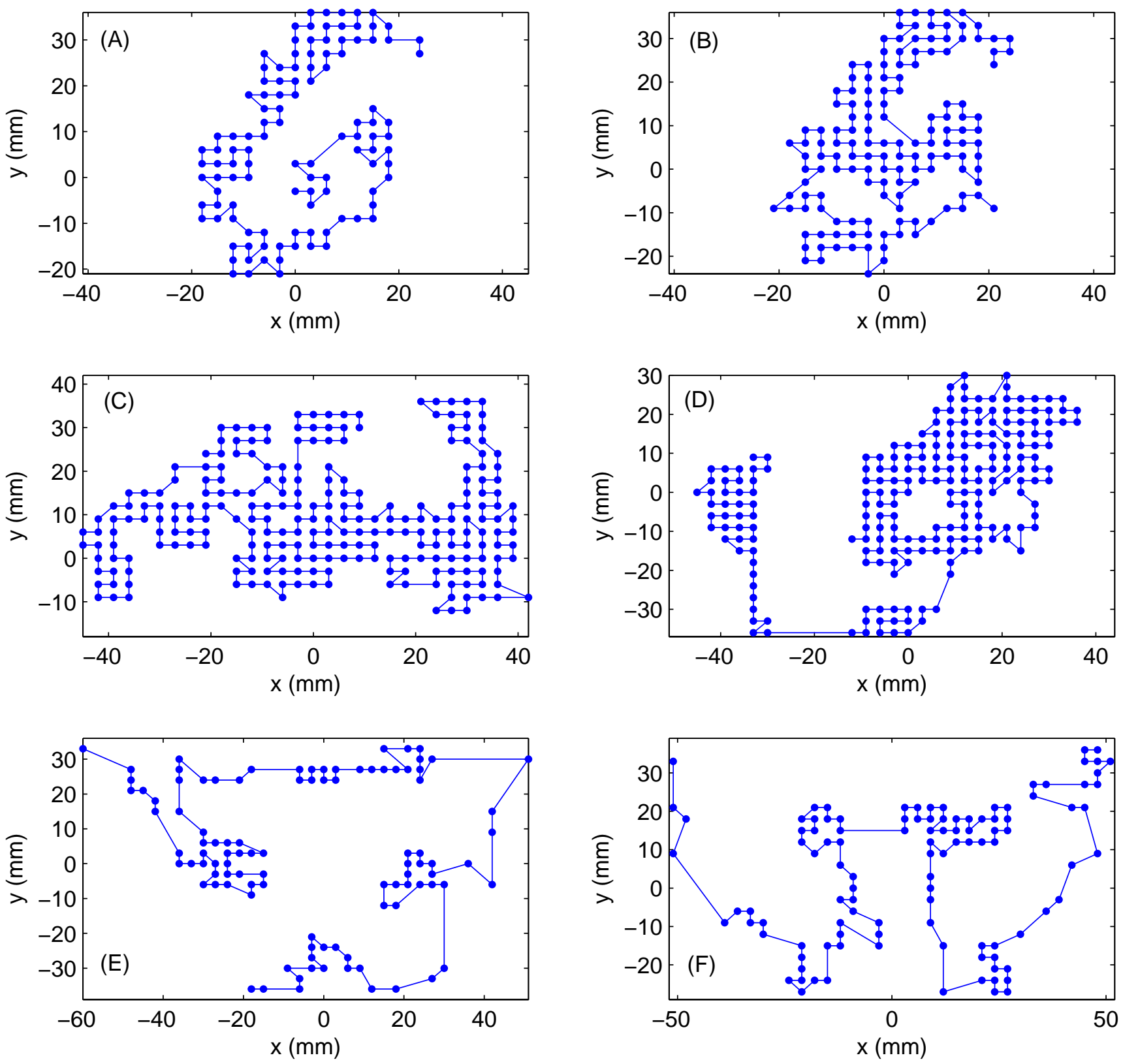

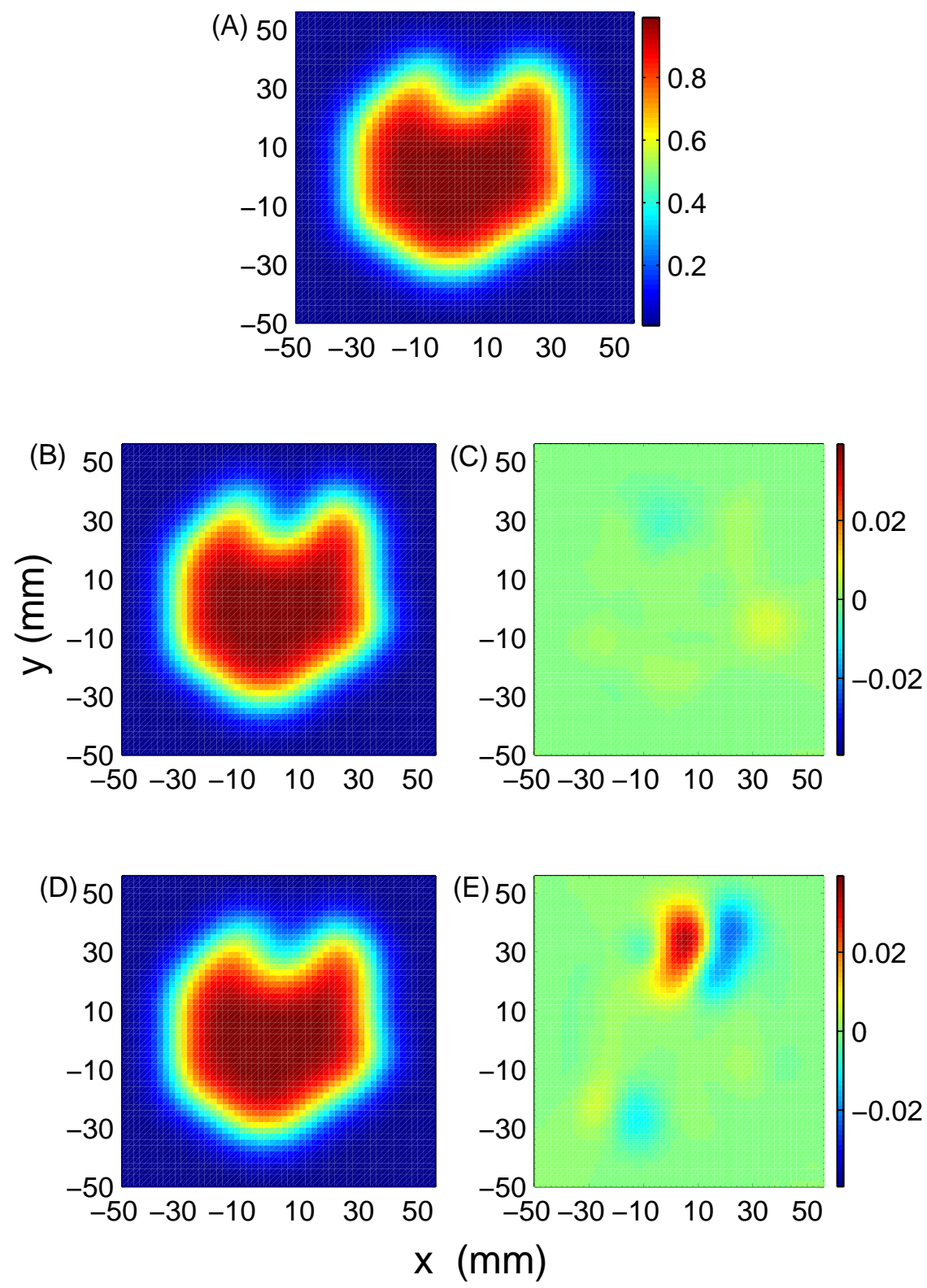


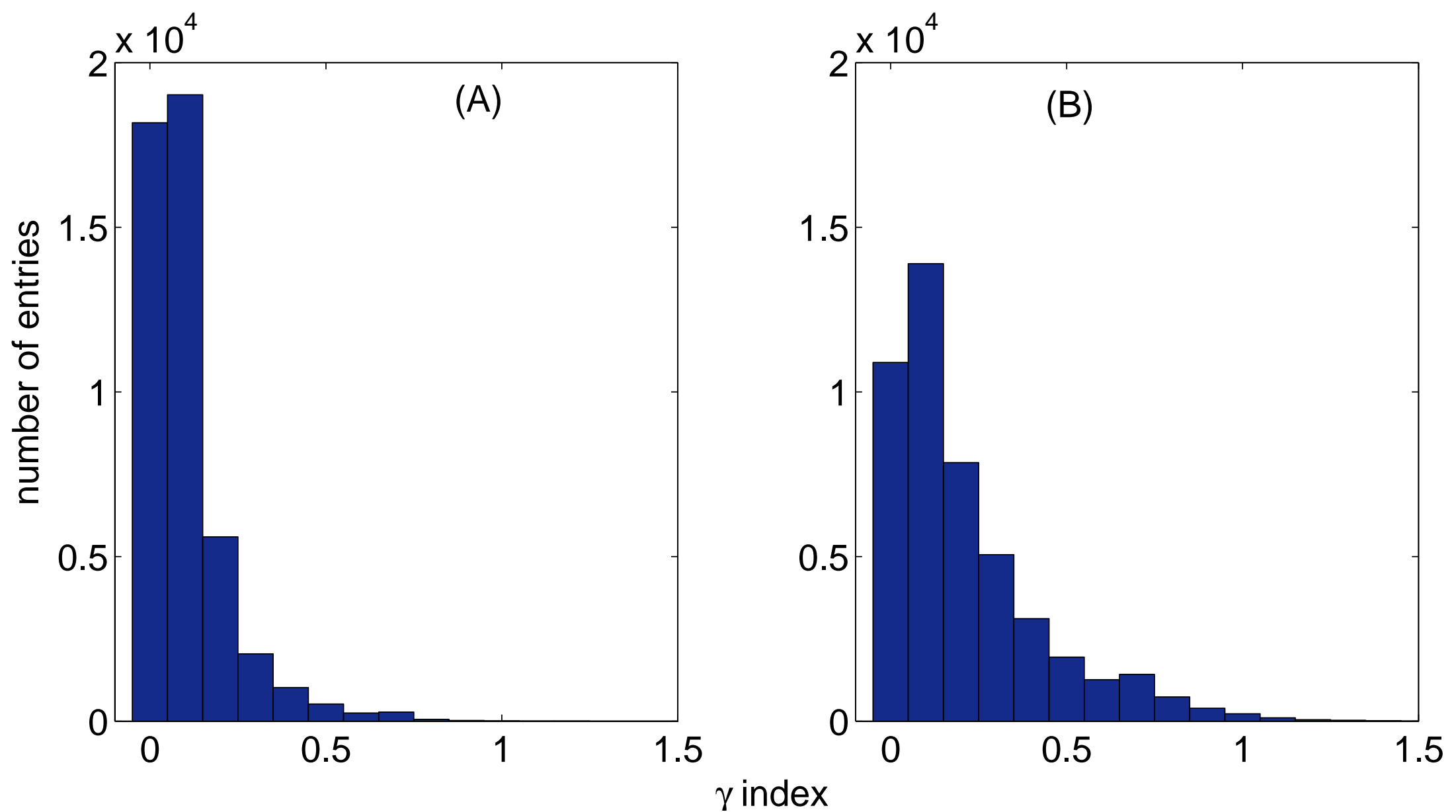

